\title{
CONJUGATE NETS IN ASYMPTOTIC PARAMETERS
}

\author{
JANET MacDONALD
}

1. Introduction. Conjugate nets on an analytic nonruled surface in ordinary space may be studied either in conjugate parameters or in asymptotic parameters. The purpose of this paper is to make some contributions to the theory of conjugate nets in asymptotic parameters.

M. L. MacQueen has studied the pencil of quadrics each of which has second-order contact with a surface at a point and third-order contact with both curves of a conjugate net at the point. This pencil of quadrics is contained in the bundle of quadrics each of which has contact of at least the third order with both curves of a conjugate net at a point. The equation of this bundle of quadrics is deduced and the bundle is studied in this paper.

W. M. Davis in his Chicago doctoral dissertation, Contributions to the theory of conjugate nets, defined and studied several canonical configurations, considering the conjugate net as parametric. This paper presents some of Davis's results translated into asymptotic parameters.

A summary of that portion of the theory of conjugate nets in asymptotic parameters which is used in subsequent sections is given in $\$ 2$. $\$ 3$ deals with the bundle of quadrics mentioned above. Certain polar relations with respect to the quadrics of this bundle are presented in $\$ 4$. In $\$ 5$ certain loci and envelopes which arise in the study of a pencil of conjugate nets at a point on a surface are studied. $\S 6$ presents a study of Davis's canonical configurations in asymptotic parameters.

2. Analytic basis. The purpose of this section is to summarize ${ }^{1}$ for later use portions of the theory of conjugate nets on an analytic nonruled surface referred to its asymptotic net in ordinary space.

Let the projective homogeneous coordinates $x_{1}, \cdots, x_{4}$ of a point $P_{x}$ on a nonruled surface $S$ in ordinary space be given by the parametric vector equation

$$
x=x(u, v) .
$$

A necessary and sufficient condition that the asymptotic net be para1944.

Presented to the Society, November 26, 1943; received by the editors March 6,

${ }^{1}$ E. P. Lane, $A$ treatise on projective differential geometry, Chicago, 1942, pp. 89, 105-113, 115-120, 125-129, 150-152, 180-190. 
metric is that the point $P_{x}$ satisfy two partial differential equations of the form

$$
\begin{aligned}
x_{u u} & =p x+\theta_{u} x_{u}+\beta x_{v}, \\
x_{v v} & =q x+\gamma x_{u}+\theta_{v} x_{v},
\end{aligned} \quad(\theta=\log \beta \gamma) .
$$

The coefficients are functions of $u, v$ which are not arbitrary but must satisfy certain integrability conditions which need not be given here.

By means of equations (2.2) the third derivatives of $x$ may be expressed as linear combinations of $x, x_{u}, x_{v}$, and $x_{u v}$.

A conjugate net $N_{\lambda}$ on an integral surface of equations (2.2) can be represented by a curvilinear differential equation of the form

$$
d v^{2}-\lambda^{2} d u^{2}=0 \quad(\lambda \neq 0),
$$

where $\lambda$ is a function of $u, v$. The two curves of the net $N_{\lambda}$ through the point $P_{x}$ will be denoted by $C_{\lambda}$ and $C_{-\lambda}$ according as $d u / d v$ has value $\lambda$ or $-\lambda$.

By the usual method, power-series expansions for the local coordinates of a point $X$ sufficiently near a fixed point $P_{x}$ and on the curve $C_{\lambda}$ through the point $P_{x}$ may be obtained:

$$
\begin{aligned}
x_{1}= & 1+2^{-1}\left(p+q \lambda^{2}\right) \Delta u^{2}+6^{-1}\left[\left(p_{u}+p \theta_{u}\right)+3\left(p_{v}+\beta q\right) \lambda\right. \\
& \left.+3\left(q_{u}+\gamma p\right) \lambda^{2}+\left(q_{v}+q \theta_{v}\right) \lambda^{3}+3 q \lambda \lambda^{\prime}\right] \Delta u^{3}+\cdots, \\
x_{2}= & \Delta u+2^{-1}\left(\theta_{u}+\gamma \lambda^{2}\right) \Delta u^{2}+6^{-1}\left[\left(p+\theta_{u}^{2}+\theta_{u u}\right)+3 \kappa \lambda\right. \\
& \left.+3 \lambda^{2}+\left(\gamma_{v}+\gamma \theta_{v}\right) \lambda^{3}+3 \gamma \lambda \lambda^{\prime}\right] \Delta u^{3}+\cdots, \\
x_{3}= & \lambda \Delta u+2^{-1}\left(\beta+\theta_{v} \lambda^{2}+\lambda^{\prime}\right) \Delta u^{2}+6^{-1}\left[\left(\beta_{u}+\beta \theta_{u}\right)+3 \pi \lambda\right. \\
& \left.+3 \kappa \lambda^{2}+\left(q+\theta_{v}^{2}+\theta_{v v}\right) \lambda^{3}+3 \theta_{v} \lambda \lambda^{\prime}+\lambda^{\prime \prime}\right] \Delta u^{3}+\cdots, \\
x_{4}= & \lambda \Delta u^{2}+6^{-1}\left(\beta+3 \theta_{u} \lambda+3 \theta_{v} \lambda^{2}+\gamma \lambda^{3}+3 \lambda^{\prime}\right) \Delta u^{3}+\cdots,
\end{aligned}
$$

where

$$
\begin{aligned}
& \lambda^{\prime}=\lambda_{u}+\lambda \lambda_{v}, \quad \kappa=\beta \gamma+\theta_{u v}, \\
& \pi=p+\beta_{v}+\beta \theta_{v}, \quad \chi=q+\gamma_{u}+\gamma \theta_{u} .
\end{aligned}
$$

The power series expansions for $C_{-\lambda}$ are obtained from these by first replacing $\lambda^{\prime}$ by $\lambda_{u}+\lambda \lambda_{v}$ and then changing the sign of $\lambda$.

The equation of the associate conjugate net is known to be

$$
d v^{2}+\lambda^{2} d u^{2}=0 .
$$

Power series expansions for a point $X$ on a curve of the associate conjugate net are obtained from equations (2.4) by changing the sign of $\lambda^{2}$. 
The equation of the pencil of conjugate nets determined by the net (2.3) is

$$
d v^{2}-\lambda^{2} h^{2} d u^{2}=0,
$$

where $h$ is a constant independent of $u$ and $v$.

Two types of congruences, $\Gamma_{1}$ and $\Gamma_{2}$, will be referred to in this paper. A line $l_{1}$ at a point $P_{x}$ on a surface ${ }^{2}$ may be regarded as determined by the points $x(1,0,0,0)$ and $y(0,-a,-b, 1)$ and also by the planes

$$
x_{3}+b x_{4}=0, \quad x_{2}+a x_{4}=0 .
$$

If $a$ and $b$ are functions of $u$ and $v$, the lines $l_{1}$ constitute a congruence $\Gamma_{1}$. The reciprocal line $l_{2}$ is the polar line of the line $l_{1}$ with respect to the quadric of Lie and may be regarded as determined by the points $\rho(-b, 1,0,0)$ and $\sigma(-a, 0,1,0)$ and also by the planes

$$
x_{4}=0, \quad x_{1}+b x_{2}+a x_{3}=0 .
$$

As in the case of a line $l_{1}$, if $a$ and $b$ are functions of $u$ and $v$, the lines $l_{2}$ constitute a congruence $\Gamma_{2}$.

The axis at a point $P_{x}$ of the conjugate net $N_{\lambda}$ is a line $l_{1}$ for which $a$ and $b$ are denoted throughout this paper by $a_{a}$ and $b_{a}$, respectively, and so are given by the formulas

$$
a_{a}=2^{-1}\left(\theta_{v}+\lambda_{v} / \lambda+\beta / \lambda^{2}\right), \quad b_{a}=2^{-1}\left(\theta_{u}-\lambda_{u} / \lambda+\gamma \lambda^{2}\right) .
$$

The ray at a point $P_{x}$ of the conjugate net $N_{\lambda}$ is a line $l_{2}$ for which $a$ and $b$ are hereinafter denoted by $a_{r}$ and $b_{r}$, respectively, and are given by the formulas

$$
a_{r}=2^{-1}\left(\theta_{v}+\lambda_{v} / \lambda-\beta / \lambda^{2}\right), \quad b_{r}=2^{-1}\left(\theta_{u}-\lambda_{u} / \lambda-\gamma \lambda^{2}\right) .
$$

The principal lines of the two curves of the conjugate net $N_{\lambda}$ at a point $P_{x}$ are the associate conjugate tangents and have the equations

$$
x_{4}=0, \quad x_{3}^{2}+\lambda^{2} x_{2}^{2}=0 .
$$

The principal join is a line $l_{2}$ for which $a$ and $b$ are hereinafter denoted by $a_{j}$ and $b_{j}$, respectively, and are given by the formulas

$$
a_{j}=2^{-1}\left(\theta_{v}+\lambda_{v} / \lambda+5 \beta / 3 \lambda^{2}\right), \quad b_{j}=2^{-1}\left(\theta_{u}-\lambda_{u} / \lambda+5 \gamma \lambda^{2} / 3\right) .
$$

The points

$$
\left(-b_{j}-i a_{j} \lambda, 1, i \lambda, 0\right), \quad\left(-b_{j}+i a_{j} \lambda, 1,-i \lambda, 0\right)
$$

are the principal points.

2 Ibid., pp. 150-152. 
The equations of the ray-point cubic at a point of the conjugate net $N_{\lambda}$ are

$$
l x_{2} x_{3}-\beta x_{2}^{3}-\gamma x_{3}^{3}=0, \quad x_{4}=0,
$$

where $l$ is defined by

$$
l=2 x_{1}+\left(\theta_{u}-\lambda_{u} / \lambda\right) x_{2}+\left(\theta_{v}+\lambda_{v} / \lambda\right) x_{3} .
$$

The equations of the ray conic determined by the conjugate net $N_{\lambda}$ at the point $P_{x}$ are

$$
4 \beta \gamma x_{2} x_{3}-l^{2}=0, \quad x_{4}=0 .
$$

3. Bundle of quadrics. This section is devoted to deducing the equation of the bundle (linear two-parameter family) of quadrics each of which has contact of at least the third order with the curves $C_{\lambda}$ and $C_{-\lambda}$ at the point $P_{x}$ and to finding all the cones in the bundle.

If the general quadric, whose equation is

$$
\begin{array}{r}
A x_{1}^{2}+B x_{2}^{2}+C x_{3}^{2}+D x_{4}^{2}+E x_{1} x_{2}+F x_{1} x_{3}+G x_{1} x_{4} \\
+H x_{2} x_{3}+I x_{2} x_{4}+J x_{3} x_{4}=0
\end{array}
$$

is to pass through the point $P_{x}(1,0,0,0)$, the coefficient $A$ must be zero. Contact of at least the third order with the curves $C_{\lambda}$ and $C_{-\lambda}$ at the point $P_{x}$ may be imposed by demanding that equation (3.1) be identically satisfied as far as the terms in $\Delta u^{3}$ by the power-series expansions for the curve $C_{\lambda}$ given in equations (2.4) and also by the corresponding expansions for the curve $C_{-\lambda}$. After this has been done and certain notational changes which need not be explicitly stated here have been made, equation (3.1) reduces to

$$
\begin{aligned}
& A\left(x_{2} x_{3}-x_{1} x_{4}-3^{-1} \gamma \lambda^{2} x_{2} x_{4}-3^{-1}\left(\beta / \lambda^{2}\right) x_{3} x_{4}\right) \\
& +B\left(2 b_{a} x_{3} x_{4}-2 \lambda^{2} a_{a} x_{2} x_{4}-\lambda^{2} x_{2}^{2}+x_{3}^{2}\right)+C x_{4}^{2}=0 .
\end{aligned}
$$

Thus the following theorem is proved.

THEOREM 1. The bundle of quadrics each of which has contact of at least the third order with the curves $C_{\lambda}$ and $C_{-\lambda}$ at the point $P_{x}$ is represented by equation (3.2).

If $B=0$, equation (3.2) reduces to the equation of a pencil of quadrics having also second-order contact with the surface $S$ at $P_{x}$. This pencil of quadrics has been studied by MacQueen. ${ }^{3}$

${ }^{3}$ M. L. MacQueen, Pencils of quadrics associated with a conjugate net, Journal of the Tennessee Academy of Science vol. 15 (1940) p. 421. 
The cones in the bundle of quadrics represented by equation (3.2) are found by setting the discriminant of the left member of this equation equal to zero. The resulting equation can be reduced to

$$
A^{2}\left(A^{2}+4 B^{2} \lambda^{2}\right)=0 \text {. }
$$

Three cases arise according as $A=0, B \neq 0$, or $A \neq 0, A^{2}+4 B^{2} \lambda^{2}=0$, or $A=0, B=0$.

In the first case, when $A=0, B \neq 0$, equation (3.2) becomes

$$
B\left(x_{3}^{2}-\lambda^{2} x_{2}^{2}-2 \lambda^{2} a_{a} x_{2} x_{4}+2 b_{a} x_{3} x_{4}\right)+C x_{4}^{2}=0 .
$$

This equation represents a pencil of noncomposite cones with vertex at $(1,0,0,0)$ in case, further,

$$
B\left(\lambda^{2} a_{a}^{2}-b_{a}^{2}\right)+C \neq 0 .
$$

If the inequality (3.5) becomes an equality, equation (3.4) represents the osculating planes of the curves $C_{\lambda}$ and $C_{-\lambda}$ at the point $P_{x}$.

In the second case when $A \neq 0, A^{2}+4 B^{2} \lambda^{2}=0$, there result two pencils of cones, the equation of one of which is

$$
\begin{aligned}
& A\left[x_{2} x_{3}-x_{1} x_{4}-\left(3^{-1} \gamma \lambda^{2}+i \lambda a_{a}\right) x_{2} x_{4}\right. \\
& \left.-\left(3^{-1} \beta / \lambda^{2}-(i / \lambda) b_{a}\right) x_{3} x_{4}-2^{-1} i \lambda x_{2}^{2}+2^{-1}(i / \lambda) x_{3}^{2}\right]+C x_{4}^{2}=0 .
\end{aligned}
$$

The equation of the other pencil of cones is obtained from equation (3.6) by replacing $i$ by $-i$. The cones represented by equation (3.6) touch the tangent plane $x_{4}=0$ in one of the associate conjugate tangents. Every one of the cones of this pencil has its vertex at one of the principal points. The other pencil of cones has the same relation to the other associate conjugate tangent and the other principal point.

In the third case, when $A=0, B=0$, equation (3.2) reduces to $x_{4}^{2}=0$. Hence the tangent plane counted twice must be considered as a cone in the bundle.

The tangent plane $x_{4}=0$ intersects the most general quadric of the bundle (3.2) in two lines whose equations are

$$
x_{4}=0, \quad B x_{3}^{2}+A x_{2} x_{3}-B \lambda^{2} x_{2}^{2}=0 .
$$

The two lines will be distinct in case $A^{2}+4 B^{2} \lambda^{2} \neq 0$. The two lines of each pair separate the associate conjugate tangents harmonically. If $A=0, B \neq 0$, the lines in which the tangent plane intersects the quadric are the conjugate tangents. If $A \neq 0, B=0$, the lines are the asymptotic tangents.

4. Polar relations. The purpose of this section is to discuss certain 
polar relations which exist among the configurations studied in this paper with respect to certain quadrics of the bundle (3.2).

The equations of the polar line of a line $l_{1}$ with respect to the most general quadric of the bundle (3.2) are $x_{4}=0$ and

$$
\begin{aligned}
A\left[x_{1}+\left(b+3^{-1} \gamma \lambda^{2}\right) x_{2}\right. & \left.+\left(a+3^{-1} \beta / \lambda^{2}\right) x_{8}\right] \\
+ & 2 B\left[\lambda^{2}\left(a_{a}-a\right) x_{2}-\left(b_{a}-b\right) x_{8}\right]=0 .
\end{aligned}
$$

This equation represents a pencil of lines with its center at the point

$$
\left[\lambda^{2}\left(a-a_{a}\right)\left(a+3^{-1} \beta / \lambda^{2}\right)+\left(b-b_{a}\right)\left(b+3^{-1} \gamma \lambda^{2}\right),\right.
$$

$$
\left.b_{a}-b, \lambda^{2}\left(a_{a}-a\right), 0\right] \text {. }
$$

Thus we prove the following theorem.

THEOREM 2. The polar lines of a line $l_{1}$ with respect to the quadrics of the bundle (3.2) form a pencil of lines in the tangent plane with the center at the point (4.2).

For the axis, equation (4.1) reduces to

$$
x_{1}+b_{j} x_{2}+a_{j} x_{3}=0 .
$$

Inspection of this equation leads to the following theorem.

TheOREM 3. At a point $P_{x}$ on the conjugate net $N_{\lambda}$ the principal join is the polar line of the axis with respect to any quadric of the bundle (3.2).

MacQueen proved ${ }^{4}$ this theorem for any quadric of the pencil of quadrics studied by him.

Elimination of the parameters $A$ and $B$ from the equations of the polar line of a line $l_{2}$ with respect to the most general quadric of the bundle (3.2) yields the equation

$$
\begin{aligned}
\lambda^{2} x_{2}^{2}+x_{3}^{2}+\left[\lambda^{2} a_{a}\right. & \left.\left(a-3^{-1} \beta / \lambda^{2}\right)+b_{a}\left(b-3^{-1} \gamma \lambda^{2}\right)\right] x_{4}^{2} \\
& +2^{-1} \lambda^{2}\left(\theta_{v}+\lambda_{v} / \lambda+3^{-1} \beta / \lambda^{2}+2 a\right) x_{2} x_{4} \\
& +2^{-1}\left(\theta_{u}-\lambda_{u} / \lambda+3^{-1} \gamma \lambda^{2}+2 b\right) x_{3} x_{4}=0 .
\end{aligned}
$$

Thus we prove the following theorem.

THEOREM 4. The locus of the polar line of a line $l_{2}$ with respect to the quadrics of the bundle (3.2) is the quadric cone represented by equation (4.4).

It is evident that this cone is intersected by the tangent plane in the associate conjugate tangents.

4 Ibid., p. 422. 
For the ray, equation (4.4) becomes

$$
\begin{aligned}
\lambda^{2} x_{2}^{2}+x_{3}^{2}+ & 4^{-1}\left[\lambda^{2}\left(\theta_{v}+\lambda_{v} / \lambda+\beta / \lambda^{2}\right)\left(\theta_{v}+\lambda_{v} / \lambda-5 \beta / 3 \lambda^{2}\right)\right. \\
& \left.+\left(\theta_{u}-\lambda_{u} / \lambda+\gamma \lambda^{2}\right)\left(\theta_{u}-\lambda_{u} / \lambda-5 \gamma \lambda^{2} / 3\right)\right] x_{4}^{2} \\
& +\lambda^{2}\left(\theta_{v}+\lambda_{v} / \lambda-3^{-1} \beta / \lambda^{2}\right) x_{2} x_{4} \\
& +\left(\theta_{u}-\lambda_{u} / \lambda-3^{-1} \gamma \lambda^{2}\right) x_{3} x_{4}=0 .
\end{aligned}
$$

Davis's canonical plane is defined ${ }^{5}$ to be the plane determined by the axis and the associate axis. The equation of this plane in asymptotic parameters is

$$
\gamma \lambda^{2} x_{2}-\left(\beta / \lambda^{2}\right) x_{3}-2^{-1}\left[\left(\beta / \lambda^{2}\right)\left(\theta_{u}-\lambda_{u} / \lambda\right)-\gamma \lambda^{2}\left(\theta_{v}+\lambda_{v} / \lambda\right)\right] x_{4}=0 .
$$

This plane intersects the cone (4.5) in the axis and in a line $l_{1}$ which is the reciprocal polar of the associate principal join. Likewise the cone which is the locus of the polar line of the associate ray with respect to the quadrics of the bundle for the associate conjugate net intersects Davis's canonical plane in the associate axis and in the reciprocal polar of the principal join.

The polar planes of a point with respect to all the quadrics of a bundle are known ${ }^{6}$ to pass through a point. The point through which pass the polar planes of a point $P_{y}$ with respect to all the quadrics of the bundle (3.2) is found by setting equal to zero the coefficients of $A, B$, and $C$ in the equation of the polar plane of $P_{v}$ with respect to the most general quadric of the bundle (3.2) and solving for the ratios of $x_{1}, \cdots, x_{4}$. Two cases arise according as $y_{4} \neq 0$ or $y_{4}=0$.

When $y_{4} \neq 0$, the resulting coordinates are given by

$$
\begin{gathered}
x_{1}=\lambda^{2} y_{2}^{2}+y_{3}^{2}-3^{-1}\left(\gamma \lambda^{2} b_{a}+\beta a_{a}\right) y_{4}^{2} \\
+2^{-1} \lambda^{2}\left(\theta_{v}+\lambda_{v} / \lambda+3^{-1} \beta / \lambda^{2}\right) y_{2} y_{4} \\
\\
+2^{-1}\left(\theta_{u}-\lambda_{u} / \lambda+3^{-1} \gamma \lambda^{2}\right) y_{3} y_{4}, \\
x_{2}=y_{4}\left(y_{3}+b_{a} y_{4}\right), \quad x_{3}=\lambda^{2} y_{4}\left(y_{2}+a_{a} y_{4}\right), \quad x_{4}=0 .
\end{gathered}
$$

Hence, for a point $P_{y}$ not in the tangent plane the polar planes with respect to all quadrics of the bundle (3.2) pass through a point in the tangent plane with coordinates (4.7). If the point $P_{y}$ lies on the axis, the point (4.7) is indeterminate and all the polar planes pass through the principal join.

- W. M. Davis, Contributions to the theory of conjugate nets, doctoral dissertation, Chicago, 1932, p. 18. 171.

- Virgil Snyder and C. H. Sisam, Analytic geometry of space, New York, 1914, p. 
When $y_{4}=0$, that is, when $P_{y}$ (supposed distinct from the point $P_{x}$ ) lies in the tangent plane, the point through which pass the polar planes of $P_{y}$ with respect to all the quadrics of the bundle (3.2) is indeterminate. The polar planes form a pencil whose axis may be represented by the equations

$$
\begin{array}{r}
y_{3} x_{2}+y_{2} x_{3}+\left(y_{1}+3^{-1} \gamma \lambda^{2} y_{2}+3^{-1}\left(\beta / \lambda^{2}\right) y_{3}\right) x_{4}=0, \\
\lambda^{2} y_{2} x_{2}-y_{3} x_{3}+\left(\lambda^{2} a_{a} y_{2}-b_{a} y_{3}\right) x_{4}=0 .
\end{array}
$$

If the point $P_{y}$ lies on one of the associate conjugate tangents, the line (4.8) is the other associate conjugate tangent. If the point $P_{v}$ coincides with $P_{x}$, the polar plane with respect to any quadric of the bundle is the tangent plane $x_{4}=0$.

5. Theory of a pencil of conjugate nets. This section deals with certain loci and envelopes which may be defined at a point of the surface in connection with a pencil of conjugate nets. The locus of the principal points, the envelope of the principal join, the envelope of the cone (4.5), the envelope of any particular one of the quadrics of the bundle (3.2), the envelope of Davis's canonical plane, the locus of Davis's canonical point, and the envelope of Davis's canonical quadric for nets of the pencil are studied.

The coordinates of one of the principal points for a general net of the pencil (2.6) are

$$
\begin{aligned}
x_{1}= & -2^{-1}\left(\theta_{u}-\lambda_{u} / \lambda+5 \gamma \lambda^{2} h^{2} / 3\right) \\
& -2^{-1} i \lambda h\left(\theta_{v}+\lambda_{v} / \lambda+5 \beta / 3 \lambda^{2} h^{2}\right), \\
x_{2}= & 1, \quad x_{3}=i \lambda h, \quad x_{4}=0 .
\end{aligned}
$$

Homogeneous elimination of $h$ leads to the equations

$$
x_{4}=0, \quad l x_{2} x_{3}-(5 / 3)\left(\beta x_{2}^{3}+\gamma x_{3}^{3}\right)=0,
$$

where $l$ is defined by equation (2.15). The same equation is obtained if the other principal point is used. Equations (5.2) represent a cubic, which will be called the principal cubic. This result may be stated in the following theorem.

TheOREM 5. At a point on a surface the locus of the principal points of all the curves of a pencil (2.6) of conjugate nets that pass through the point is the principal cubic of the pencil, represented by equations (5.2).

Comparison of equations (5.2) with equations (2.14) shows that the principal cubic and the ray-point cubic are in the pencil of cubics, in the tangent plane, with equations $x_{4}=0$ and 


$$
l x_{2} x_{3}-k\left(\beta x_{2}^{3}+\gamma x_{3}^{3}\right)=0,
$$

where $k$ is an arbitrary parameter. The principal cubic is characterized by $k=5 / 3$, and the ray-point cubic by $k=1$.

The principal join for a general net of the pencil (2.6) is represented by the equations $x_{4}=0$ and

$$
\begin{aligned}
x_{1} & +2^{-1}\left(\theta_{u}-\lambda_{u} / \lambda+5 \gamma \lambda^{2} h^{2} / 3\right) x_{2} \\
& +2^{-1}\left(\theta_{v}+\lambda_{v} / \lambda+5 \beta / 3 \lambda^{2} h^{2}\right) x_{3}=0 .
\end{aligned}
$$

Application of the theory of envelopes leads to the equations

$$
x_{4}=0, \quad 100 \beta \gamma x_{2} x_{3} / 9-l^{2}=0 .
$$

This equation represents a conic, which will be called the principal conic of the pencil (2.6) of conjugate nets at the point $P_{x}$. This result may be stated in the following theorem.

THEOREM 6. At a point on a surface the envelope of the principal joins of all the nets of a pencil (2.6) of conjugate nets is the principal conic, represented by equations (5.5).

Comparison of equations (5.5) with equations (2.16) shows that the principal conic and the ray conic are in the pencil of conics, in the tangent plane, with equations

$$
x_{4}=0, \quad 4 k \beta \gamma x_{2} x_{3}-l^{2}=0,
$$

where $k$ is an arbitrary parameter. The principal conic is characterized by $k=25 / 9$, and the ray conic by $k=1$.

Replacing $\lambda$ by $\lambda h$ in equation (4.5) and applying the theory of envelopes leads to the following equation of the envelope of the cone (4.5) for the pencil of conjugate nets (2.6):

$$
\begin{array}{r}
5 \beta^{2} x_{4}^{2} m^{3} / 3+m^{2} n^{2}+5 \gamma^{2} x_{4}^{2} n^{3} / 3+25 \beta^{2} \gamma^{2} x_{4}^{4} m n / 8 \\
-625 \beta^{4} \gamma^{4} x_{4}^{8} / 768=0,
\end{array}
$$

where $m$ and $n$ are polynomials in $x_{2}, x_{3}$, and $x_{4}$ of degree four. Hence the envelope of the cone (4.5) is a surface of order eight.

Application of the theory of envelopes to the equation of any particular one of the quadrics of the bundle (3.2) leads to the following equation:

$$
\begin{aligned}
& \left(4 A \beta x_{3} x_{4} / 9\right)\left\{x_{2} x_{4}\left[A \gamma+3 B\left(\theta_{v}+\lambda_{v} / \lambda\right)\right]+3 B x_{2}^{2}-3 B \gamma x_{3} x_{4}\right\} \\
& =\left\{A\left(x_{2} x_{3}-x_{1} x_{4}\right)+B\left[x_{3}^{2}-\beta x_{2} x_{4}\right.\right. \\
& \left.\left.\quad+\left(\theta_{u}-\lambda_{u} / \lambda\right) x_{3} x_{4}\right]+C x_{4}^{2}\right\}^{2}
\end{aligned}
$$


Hence, the envelope of any particular one of the quadrics of the bundle (3.2) is a quartic surface (5.8).

For a general net of the pencil (2.6) of conjugate nets the equation of Davis's canonical plane is

$$
\begin{aligned}
\gamma \lambda^{2} h^{2} x_{2}-\left(\beta / \lambda^{2} h^{2}\right) x_{3}-2^{-1}\left[\left(\beta / \lambda^{2} h^{2}\right)\right. & \left(\theta_{u}-\lambda_{u} / \lambda\right) \\
& \left.-\gamma \lambda^{2} h^{2}\left(\theta_{v}+\lambda_{v} / \lambda\right)\right] x_{4}=0 .
\end{aligned}
$$

Inspection shows that this plane passes through the cusp-axis,

$$
2 x_{3}+\left(\theta_{u}-\lambda_{u} / \lambda\right) x_{4}=0, \quad 2 x_{2}+\left(\theta_{v}+\lambda_{v} / \lambda\right) x_{4}=0 .
$$

Thus we prove the following theorem.

Theorem 7. At a point on the surface Davis's canonical planes with respect to all the nets of the pencil (2.6) form a pencil of planes with the cusp-axis as axis.

For a general net of the pencil (2.6) the coordinates of Davis's canonical point, ${ }^{7}$ the intersection of the ray and the associate ray, are given by

$$
\begin{aligned}
& x_{1}=\left(2^{-1} \beta / \lambda^{2} h^{2}\right)\left(\theta_{u}-\lambda_{u} / \lambda\right)-2^{-1} \gamma \lambda^{2} h^{2}\left(\theta_{v}+\lambda_{v} / \lambda\right), \\
& x_{2}=-\beta / \lambda^{2} h^{2}, \quad x_{3}=\gamma \lambda^{2} h^{2}, \quad x_{4}=0 .
\end{aligned}
$$

Homogeneous elimination of the parameter $h$ yields the equations of the flex-ray:

$$
x_{4}=0, \quad x_{1}+2^{-1}\left(\theta_{u}-\lambda_{u} / \lambda\right) x_{2}+2^{-1}\left(\theta_{v}+\lambda_{v} / \lambda\right) x_{3}=0 .
$$

Thus we prove the theorem.

THEOREM 8. At a point on a surface the locus of Davis's canonical point with respect to a pencil (2.6) of conjugate nets on the surface is the flex-ray of the pencil of conjugate nets.

Davis's canonical quadric at a point $P_{x}$ of a conjugate net is defined $^{8}$ as the unique quadric surface having the following properties:

1. The quadric has second-order contact with the surface sustaining the net.

2. The axis and the ray of the net at $P_{x}$ are reciprocal polars with respect to the quadric.

3. The quadric passes through the point $P_{y}$ which is the harmonic conjugate of $P_{x}$ with respect to the focal points of the axis.

${ }^{7}$ Davis, op. cit., p. 17.

8 Ibid., p. 11. 
The equation of Davis's canonical quadric at a point $P_{x}$ of a general net of the pencil (2.6) is

$$
\begin{aligned}
x_{2} x_{3}-x_{1} x_{4} & +\gamma \lambda^{2} h^{2} x_{2} x_{4}+\left(\beta / \lambda^{2} h^{2}\right) x_{3} x_{4} \\
& +\left[-2^{-1} \theta_{u v}+4^{-1} \gamma_{v} \lambda^{2} h^{2}+4^{-1} \beta_{u} / \lambda^{2} h^{2}\right. \\
& +2^{-1} \gamma \lambda^{2} h^{2}\left(\theta_{v}+2 \lambda_{v} / \lambda\right) \\
& \left.+\left(2^{-1} \beta / \lambda^{2} h^{2}\right)\left(\theta_{u}-2 \lambda_{u} / \lambda\right)\right] x_{4}^{2}=0 .
\end{aligned}
$$

Application of the theory of envelopes yields an equation of a quartic surface as the equation of the envelope of Davis's canonical quadrics for all the nets of a pencil (2.6) of conjugate nets.

6. Canonical configurations. In W. M. Davis's dissertation, Contributions to the theory of conjugate nets, the conjugate net is considered as parametric. The purpose of this section is to exhibit some of Davis's results in asymptotic parameters and to show some relationships that exist among the configurations studied by him. The canonical configurations referred to are those defined ${ }^{9}$ by Davis.

The equation of Davis's canonical quadric for the net $N_{\lambda}$ may be obtained from equation (5.13) by setting $h=1$. The following equation of his associate canonical quadric may be obtained from the same equation (5.13) by setting $h=1$ and replacing $\lambda^{2}$ by $-\lambda^{2}$ :

$$
\begin{aligned}
& x_{2} x_{3}-x_{1} x_{4}-\gamma \lambda^{2} x_{2} x_{4}-\left(\beta / \lambda^{2}\right) x_{3} x_{4} \\
& +\left[-2^{-1} \theta_{u v}-4^{-1} \gamma_{v} \lambda^{2}-4^{-1} \beta_{u} / \lambda^{2}-2^{-1} \gamma \lambda^{2}\left(\theta_{v}+2 \lambda_{v} / \lambda\right)\right. \\
& \left.-\left(2^{-1} \beta / \lambda^{2}\right)\left(\theta_{u}-2 \lambda_{u} / \lambda\right)\right] x_{4}^{2}=0 .
\end{aligned}
$$

If the equations of Davis's canonical quadric and associate canonical quadric are added, and if the result is divided by 2 , the equation of the quadric of Wilczynski is obtained:

$$
x_{2} x_{3}-x_{1} x_{4}-2^{-1} \theta_{u v} x_{4}^{2}=0 .
$$

Hence the quadric of Wilczynski belongs to the pencil of quadrics determined by Davis's canonical quadric and associate canonical quadric.

The coordinates of Davis's canonical point for the net $N_{\lambda}$ are given by

$$
\begin{array}{lr}
x_{1}=\left(2^{-1} \beta / \lambda^{2}\right)\left(\theta_{u}-\lambda_{u} / \lambda\right)-2^{-1} \gamma \lambda^{2}\left(\theta_{v}+\lambda_{v} / \lambda\right), \\
x_{2}=-\beta / \lambda^{2}, \quad x_{3}=\gamma \lambda^{2},
\end{array}
$$

A little computation shows that the canonical point (6.3) and the

' Ibid., pp. 17-25. 
canonical plane (4.6) are pole and polar with respect to both the canonical quadric and the associate canonical quadric.

The pencil of lines in the canonical plane with center at $P_{x}$ is defined by Davis to be the first canonical pencil of the net, and the pencil of lines in the tangent plane at $P_{x}$ with center at the canonical point is defined to be the second canonical pencil of the net. The flexray, represented by equations (5.12), belongs to the second canonical pencil.

The intersection of the tangent plane with the canonical plane is defined by Davis to be the first canonical tangent. The equations of the first canonical tangent in asymptotic parameters are $x_{4}=0$ and

$$
\gamma \lambda^{2} x_{2}-\left(\beta / \lambda^{2}\right) x_{3}=0 .
$$

The line joining the canonical point to the point $P_{x}$ is defined by Davis to be the second canonical tangent. The equations of this line are $x_{4}=0$ and

$$
\gamma \lambda^{2} x_{2}+\left(\beta / \lambda^{2}\right) x_{3}=0 .
$$

Davis's thesis includes a study of congruences of pairs of lines which are polar lines with respect to his canonical quadric at a point of a conjugate net of a surface, when one of the lines, $l_{1}$, passes through $P_{x}$ but does not lie in the tangent plane, and the other line, $l_{2}$, lies in the tangent plane but does not pass through $P_{x}$. Only such reciprocal polar lines as belong to Davis's canonical pencils are considered.

As in $\$ 2$, a line $l_{1}$ may be represented by equations

$$
x_{2}+a x_{4}=0, \quad x_{3}+b x_{4}=0 .
$$

The line $l_{2}$ which is the reciprocal polar of $l_{1}$ with respect to Davis's canonical quadric may be represented by equations $x_{4}=0$ and

$$
x_{1}+\left(b-\gamma \lambda^{2}\right) x_{2}+\left(a-\beta / \lambda^{2}\right) x_{3}=0 .
$$

The lines $l_{1}$ and $l_{2}$ will be lines of the first and the second canonical pencils (in the sense of Davis), respectively, if

$$
a=2^{-1}\left(\theta_{v}+\lambda_{v} / \lambda+h \beta / \lambda^{2}\right), \quad b=2^{-1}\left(\theta_{u}-\lambda_{u} / \lambda+h \gamma \lambda^{2}\right),
$$

where $h$ is a parameter.

If $h=1$, then $a=a_{a}, b=b_{a}$, and $a-\beta / \lambda^{2}=a_{r}, b-\gamma \lambda^{2}=b_{r}$. Hence, for $h=1$, the line $l_{1}$ is the axis and $l_{2}$ the ray of the conjugate net $N_{\lambda}$. If $h=-1$, then $l_{1}$ is the associate axis. If $h=3$, then $l_{2}$ is the associate ray. If $h=2$, then $l_{2}$ is the flex-ray. If $h=0$, then $l_{1}$ is the cusp-axis. If $h=\infty$, the lines $l_{1}$ and $l_{2}$ are Davis's first and second canonical tangents, respectively. Any pair of lines $l_{1}$ and $l_{2}$ given by the same 
value of $h$ in (6.8) are reciprocal polars with respect to Davis's canonical quadric. It is seen that the associate axis and the associate ray are not corresponding lines. Likewise, the flex-ray and the cusp-axis are not corresponding lines.

Davis defines at each point of a surface three tangents analogous to the tangents of Darboux. All the quadric surfaces represented by the equation

$$
x_{2} x_{3}-x_{1} x_{4}+\gamma \lambda^{2} x_{2} x_{4}+\left(\beta / \lambda^{2}\right) x_{3} x_{4}+k_{4} x_{4}^{2}=0
$$

have second order contact with the surface at $P_{x}$ and intersect the surface in a curve with a triple point at $P_{x}$. The triple point tangents of this curve are defined to be the triple tangents of the conjugate net at the point $P_{x}$. They can be shown to intersect the ray-point cubic (2.14) in three collinear points lying on a line in Davis's second canonical pencil, defined by Davis to be the line of collineation of the net $N_{\lambda}$. The associate line of collineation is the reciprocal polar of the associate axis with respect to Davis's canonical quadric.

In addition to the lines already mentioned, the principal join and the associate principal join are also members of Davis's second canonical pencil, the principal join being characterized by $h=11 / 3$, and the associate principal join by $h=1 / 3$.

University of Chicago 